\title{
Smart Textile: Exploration of Wireless Sensing Capabilities
}

\author{
Andrey Somov \\ CDISE \\ Skolkovo Institute of Science and Technology \\ Moscow, Russia \\ a.somov@skoltech.ru
}

\author{
Elias Torres Alonso, Monica F. Craciun, \\ Ana I. S. Neves, Anna Baldycheva \\ CEMPS \\ University of Exeter, UK \\ Exeter, UK \\ \{m.f.craciun, a.neves, a.baldycheva\}@exeter.ac.uk
}

\begin{abstract}
E-textile is a developing technology joining the advantages of material science and information and communication technologies. In this work, we present the development and assessment of smart textile system containing sensing, processing and wireless communication capabilities. We demonstrate a wearable temperature sensing system based on resistance temperature detection approach utilizing graphene technology, which allows high flexibility and robustness of the electronic textile. The developed sensing system demonstrates experimental sensitivity as high as $80 \Omega /{ }^{\circ} \mathrm{C}$ within the temperature detection range from $24^{\circ} \mathrm{C}$ to $35^{\circ} \mathrm{C}$, which is the highest reported to date for wearable temperature sensors. In terms of wireless communication, the system operates at $2.4 \mathrm{GHz}$ supporting Bluetooth low energy technology and securely transmits the measured data for up to $10 \mathrm{~m}$ which is proved by received signal strength and link quality indicators.
\end{abstract}

Keywords-e-textile; smart textile; wireless sensing, wearable temperature sensor

\section{INTRODUCTION}

Internet of Things (IoT) is an emerging technology aiming at seamless and unobtrusive communication between smart objects and services [1] and their adjustment to changing environmental or situation conditions. Wearable technology is a good example of IoT: wearables or "things" are able to talk to another local and remote "things" as well as associate the owner with a wearable sensor and context [2]. E-textile is among the pillar technologies for wearables and IoT, which focuses on a number of applications, e.g. sport, medicine, elderly people support [3].

Currently, there is a tremendous progress in terms of fabrication of sensors able to be deployed on textile [4][5][6]. At the same time there is a number of approaches reporting on integration of electronics in textile: e-stripes and conductive threads [7] and other methods [8]. However, there are just a few works available in the literature reporting on the e-textile systems able to sense, process the measured data and communicate them to a remote user or operator. For example, in our recent work, we presented processing of the thermistor polycarbonate/ $\alpha$ '-(BEDT-TTF $)_{2} \mathrm{I}_{\mathrm{x}} \mathrm{Br}_{3-\mathrm{x}}$ into polyester textile. It enables engineering of e-textile which can detect relatively small temperature changes [13]. For feasibility purpose we implemented the associated 'proof-of-concept' by designing a wireless sensor node with the proposed thermistor [9]. Hence, there is still a lack of multidisciplinary research which could result in the state-of-the-art e-textile systems.

In this work, we present the concept of exploring graphenecoated textile fibres [10] as vital sign sensors, for example for monitoring body temperature, fully integrated on textiles and enriched with wireless sensing option [14]. This versatile method can be performed with textile fibres of different materials and shapes, and with different types of graphene. Sheet resistance of graphene-coated textile fibres is very sensitive to external stimuli, such as temperature, strain and stress, or contact with certain chemicals, and can therefore be explored as wearable sensing devices.

\section{PlatForm Design}

In this section we present the e-textile platform with sensing, processing and wireless communication capabilities and describe its interfacing with a smartphone.

\section{A. Platform Overview}

The proposed e-textile platform includes four units: sensing, processing, wireless communication and power supply (see Fig. 1).

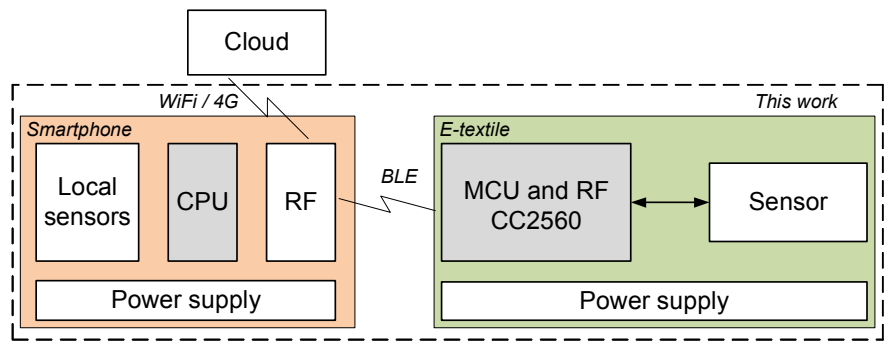

Fig. 1. Block diagram of prototyped wireless sensing system.

Processing (MCU) and wireless communication (RF) are realized using the CC2560 controller relying on Bluetooth Low Energy 4.1 (BLE) technology compatible with IEEE802.15.4 standard. This feature allows for communicating at $2.4 \mathrm{GHz}$ between the proposed e-textile and an external wireless devices supporting Bluetooth, e.g. a smartphone. This idea adds extra value to the user: the measured data can be forwarded to a cloud 
service for further powerful data processing, e.g. making inference or prediction procedures. These procedures can not be implemented on board of a low power micro controller. As a power supply we use the button type Li battery CR-2477 (3 V, $1000 \mathrm{mAh}$ ) for experimenting and proof of concept reasons. We do not investigate the power management strategy in details as it is out of scope of this work. Instead we use the available on board of CC2560 power management block. We present sensing unit in details in Section II-B.

\section{B. Sensor Design and Fabrication}

The E-textile smart temperature sensor design is based on the resistance temperature detection approach and consists of the graphene-coated polypropylene (PP) fibres (see Fig. 2). Utilising the graphene technology, we take an advantage of the sharp change in sheet resistance of the graphene PP fibres upon change of temperature and high robustness of graphene films to bending and stretching, which was demonstrated in our previous works [11][13].

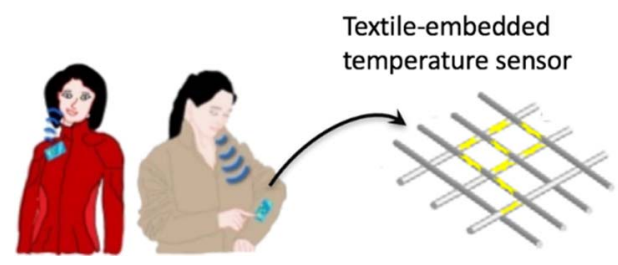

Fig. 2. Vision of wireless communication wearable sensing devices based on a system fabricated on fibers of textiles using graphene thin film technology.

The proposed sensors are prepared by coating the PP fibres with single-layer graphene which is grown by chemical vapor deposition with a cold-wall reaction chamber, using methane as a carbon source and $0.025 \mathrm{~mm}$ thick $99.999 \%$ pure copper foil. The copper foils are annealed at $1035^{\circ} \mathrm{C}$ under $H_{2}(0.4 \mathrm{sccm})$ for 10 minutes, the growth is performed under $H_{2}(2 \mathrm{sccm})$ and $\mathrm{CH}_{4}(35 \mathrm{sccm})$ for 5 minutes, and then the chamber is rapidly cooled to room temperature for 10 minutes with a large $\mathrm{Ar}$ flow $(50 \mathrm{sccm})$. The thickness and quality of the graphene film is first tested by transferring a CVD-grown graphene film onto a $\mathrm{SiO}_{2} / \mathrm{Si}$ substrate and examined by optical microscopy and Raman. This is followed by the transfer process, a thin poly(methyl metracrylate) (PMMA 950K A4) film is spincoated on top of the graphene, and submitted to $A r$ plasma to etch any extra graphene on the backside. The copper is then etched in an aqueous solution of ammonium persulfate $0.1 \mathrm{M}$, the floating graphene and PMMA sheet is washed several times with DI water. After this step, the graphene and PMMA sheet are transferred to the fibers. Finally, the PMMA is dissolved with acetone, leaving the graphene on the surface of the fibers.

To take the measurements we create metallic pads for connecting the controller device CC2560 (see Fig. 1) to the sensor. We use the controller's 12-bit Analogue-to-Digital Converter (ADC) for measuring temperature. In terms of measurements we evaluate the sensor conductivity by measuring the current flowing through the sensor.

\section{RESULTS}

In this section we present the results obtained during the experimental work with the proposed e-textile platform.

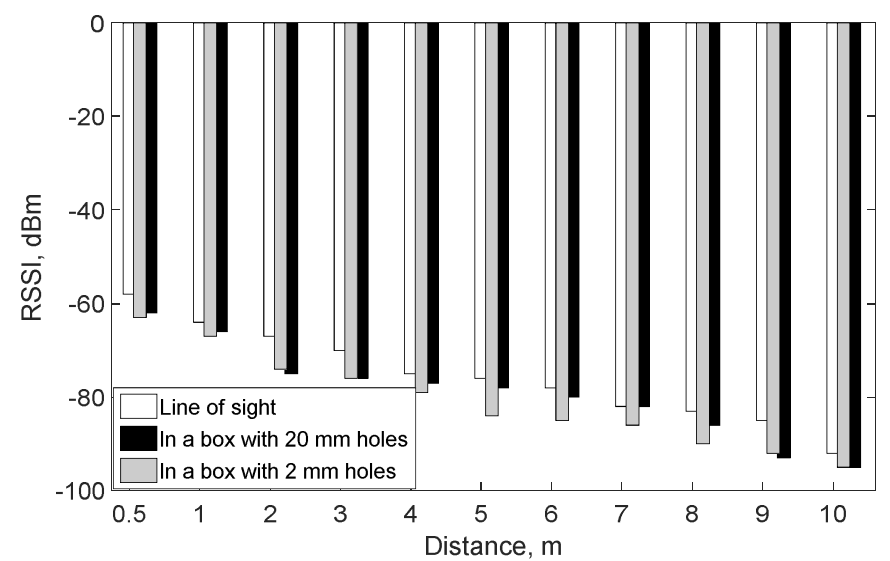

Fig. 3. Wireless link assessment using RSSI.

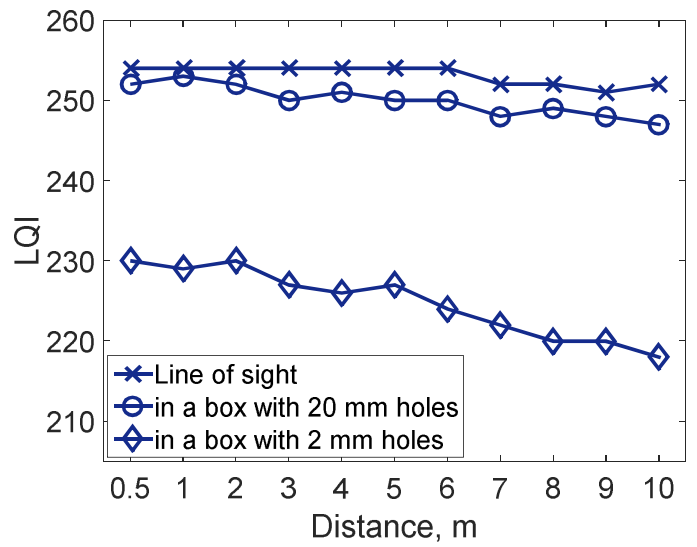

Fig. 4. Wireless link assessment using LQI.

\section{A. Assessment of Wireless Link}

In this section, we experimentally evaluate the quality of wireless link between the e-textile and smartphone. In the scope of this experiment we consider three scenarios: (i) direct line of sight communication, non direct line of sight when the smartphone is locked, for instance, in a washroom cubicle (ii) with holes of $2 \mathrm{~mm}$ diameter and (iii) $20 \mathrm{~mm}$ diameter on its front door. The assessment of wireless link between two devices is effectuated using Received Signal Strength Indicator (RSSI) and Link Quality Indicator (LQI) metrics shown in Fig. 3 and Fig. 4, respectively. This experiment demonstrates that the link with low RSSI might have high LQI and vice versa. Wireless link assessment demonstrates that the link quality does not degrade drastically within $10 \mathrm{~m}$ distance between the e-textile and smartphone supporting BLE technology (see Section II).

\section{B. Sensor Assessment}

The electric conductivity is measured by a two-probe method using tungsten probes and a Keithley 237 sourcemeasure unit [10]. We calculate the sheet resistance by subtracting the contact resistance and by taking into account the aspect ratio of the graphene section on each sample. To estimate the contact resistance we utilize a method commonly used for graphene devices [11][12], based on measuring the two-probe resistance in devices with different contact separation keeping the width of the channel constant. Fig. 5 shows the change in 
temperature (black curve) and corresponding change in sheet resistance (blue curve) of two graphene-coated polypropylene fibres, over time. The temperature detection range depends on the initial resistance of graphene film. For example, as presented in Fig 5, the resistances at $25{ }^{\circ} \mathrm{C}$ for Fiber 1 and Fiber 2 are $\sim 8670 \Omega$ and $\sim 8750 \Omega$, respectively. An increase in temperature results in reduction of resistance for both fibers down to $\sim 8550 \Omega$, while Fiber 2 reaches this minimum at $35^{\circ} \mathrm{C}$, Fiber 1 demonstrates its minimal resistance at already $30{ }^{\circ} \mathrm{C}$. Overall, the fabricated textile sensors demonstrate an average sensitivity as high as $80 \Omega /{ }^{\circ} \mathrm{C}$.

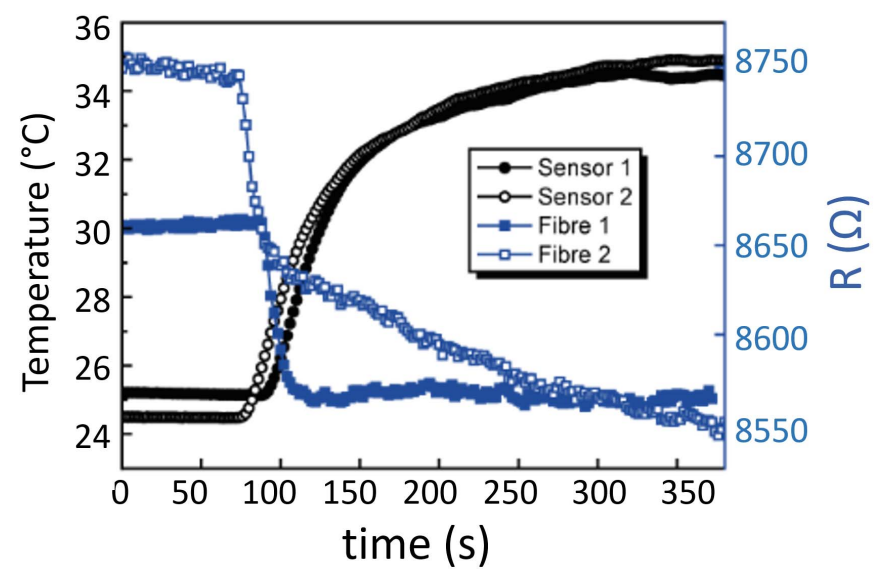

Fig. 5. Experimental demonstration of sensing capabilities (sensitivity and range of operation) of two sensors fabricated using the technology described in Section II-B, but with different initial resistance (Fiber 1 and Fiber 2).

The effect of bending on the electrical resistance of the graphene-coated PP fibers was tested for analyzing its flexibility and suitability as a true multiple use smart textile sensing system. The sheet resistance of graphene-coated PP fibers were monitored during bendings of fibers down to $5 \mathrm{~mm}$. The samples demonstrate stability upon bending for 1000 times and, for approximately one third of the samples, the sheet resistance showed little variation [new 9]. The limiting factor for the graphene textile samples seems to be the textile fiber itself, which is more fragile, and shreds upon bending, forming breaks and discontinuities on the graphene sheet which lead to debonding of graphene. However, utilizing a photo-sensitized oxidation process on the fibers prior to the graphene deposition, employing ultraviolet-ozone (UVO) treatment [10], we can reduce the variation down to a bending radius of $2.4 \mathrm{~mm}$ (approximate tensile strength of $2 \%$ ). This is due to the fact that the UVO treatment not only smoothes the textile fiber surface and increases the adhesion of graphene, but it also improves the mechanical strength of the fibers [10].

\section{DISCUSSION}

In this paper, we have presented an e-textile temperature sensing system enriched with processing and wireless communication capabilities aiming at the applications in the medical and sport domains. For the first time, we demonstrate a wearable temperature sensing system based on resistance temperature detection approach and fabricated on textile fibers using graphene technology. The developed sensing system demonstrate sensitivity as high as $80 \Omega /{ }^{\circ} \mathrm{C}$, high robustness to bending and stretching. In terms of wireless communication, the system was tested in three scenarios including direct line of site and non direct line of site conditions. In all cases the designed e-textile system is able to securely transmit the measured data for up to $10 \mathrm{~m}$ using BLE wireless communication.

Our future work includes both vital signs sensing (body temperature, heartbeat and breathing) and context aware sensing based on the mutli-plexed sensing e-textile system using the smartphone sensors. In particularly, it is also important to understand the effects of multiple sensors on the operation of the entire system, as well as to evaluate the error threshold. This idea helps to add extra value to raw sensor data measured by the sensors. The resultant data flow will be forwarded to a cloud service for creating knowledge and inference procedures out of measured data. It will be helpful for predicting health issues and suggesting a proper training load at particular day time.

\section{REFERENCES}

[1] D. Miorandi, S. Sicari, F. De Pellegrini, I. Chlamtac, "Internet of things: Vision, applications and research challenges," Ad Hoc Networks, vol. 10, no 7, pp. 1497-1516, 2012.

[2] C. C. Ching, M. K. Stewart, D. E. Hagood and R. N. Rashedi, "Representing and Reconciling Personal Data and Experience in a Wearable Technology Gaming Project," IEEE Trans. Learning Technologies, vol. 9, no. 4, pp. 342-353, Oct.-Dec., 2016.

[3] M. Stoppa, and A. Chiolerio, "Wearable Electronics and Smart Textiles: A Critical Review”, Sensors, vol. 14, pp. 11957-11992, 2014.

[4] L. M. Castano, and A. B. Flatau, "Smart Fabric sensors and e-textiles technologies: a review", Smart Mater. Struct., vol. 23, 053001 (27 pp), 2014.

[5] L. R. Ferreras, et al., "Highly piezoresistive textiles based on a soft conducting charge transfer salt," J. Mater. Chem., vol 21, pp. 637-640, 2011.

[6] Z. Cao, M. J. Tudor, R. N. Torah, S. P. Beeby, "Screen Printable Flexible BiTe-SbTe-Based Composite Thermoelectric Materials on Textiles for Wearable Applications," IEEE Trans. Electron Devices, vol. 63, no. 10, pp. 4024-4030, Oct. 2016.

[7] C. Zysset, T. W. Kinkeldei, N. Munzenrieder, K. Cherenack and G. Troster, "Integration Method for Electronics in Woven Textiles," IEEE Trans. Components, Packaging and Manufacturing Technology, vol. 2, no. 7, pp. 1107-1117, July 2012.

[8] Electronic Textiles - Smart Fabrics and Wearable Technology, T. Dias Eds., Woodhead Publishing, Oxford, 2015.

[9] V. Lebedev, et al., "Investigation of sensing capabilities of organic bilayer thermistor in wearable e-textile and wireless sensing devices," Organic Electronics, vol. 42, pp. 146-152, Mar. 2017.

[10] A. I. S. Neves, et.al., "Transparent conductive graphene textile fibres", Sci. Rep., vol. 5, 09866 (7 pp), 2015.

[11] Craciun, M.F. et al. Trilayer graphene is a semimetal with a gate-tunable band overlap. Nature Nanotech. 4, 383-388, 2009.

[12] Russo, S. et al. Contact resistance in graphene-based devices. Physica E 42, 677-679, 2010.

[13] Ana I. S. Neves, et al., "Towards conductive textiles: coating polymeric fibres with graphene," Sci. Rep., vol. 7, 04250 (1 0pp), 2017.

[14] A. Baranov, D. Spirjakin, S. Akbari, A. Somov, R. Passerone, "POCO: 'Perpetual' operation of $\mathrm{CO}$ wireless sensor node with hybrid power supply," Sensors and Actuators, vol. 238, pp. 112-121, Feb. 2016. 